\title{
Acción sin daño: un análisis de las intervenciones de una organización de la sociedad civil ambientalista en comunidades costeras del noroeste de México
}

\author{
Do no Harm: An Analysis of the Interventions of an Environmental Civil \\ Society in Coastal Communities of Northwestern, Mexico \\ Jorge Torre \\ COMUNIDAD Y BIODIVERSIDAD, A.C., jtorre@cobi.org.mx
}

\section{Francisco Fernández Rivera-Melo \\ COMUNIDAD Y BIODIVERSIDAD, A.C., ffernandez@cobi.org.mx}

En la primera parte del artículo se presenta tanto el contexto pesquero como el de las organizaciones de las sociedad civil en México a inicios del siglo xxi. En la segunda parte se hace un análisis comparativo, usando la metodología Acción Sin Daño, de cinco intervenciones realizadas por una Organización de la Sociedad Civil (osc) para lograr la implementación de reservas marinas completamente protegidas en el noroeste del país. El papel de las osc identificadas como ambientalistas ha ido cambiando significativamente en la última década, éstas trabajan en lograr un equilibrio de bienestar ambiental, socioeconómico y de gobernanza. Una de las principales trampas en que caen las osc es dejar de escuchar a las comunidades, y entrar con una actitud paternalista, lo cual muy probablemente, aun cuando nobles, harán que los proyectos fracasen. En palabras de un pescador "les gana el corazón y no la cabeza”.

PALABRAS ClAVE: comunidades costeras, osc, gobierno, pesquerías, reservas marinas.

The first part of this essay describes the settings of fisheries and civil society organizations (cso, one type of NGO) in Mexico in the early $21^{\text {st }}$ century. The second presents a comparative analysis, from the so-called "Do no Harm" perspective, of five interventions carried out by a cso to achieve the establishment of fully-protected marine reserves in northwestern Mexico. The role of csos identified as environmentalist has changed significantly in the last decade, as they work to achieve a balance among environmental health, socioeconomic well-being and good governance. One of the main pitfalls into which csos tend to stumble occurs when they cease to listen to the voices of communities and adopt paternalistic attitudes that, while perhaps noble, may cause projects to fail. In the words of one fisherman: "their heart wins, not their head".

KEYwORDs: coastal communities, NGO, government, fisheries, marine reserves.

Fecha de recepción: 24 de noviembre de 2016 / Fecha de aprobación: 26 de julio de 2017 / Fecha de recepción de la versión final: 10 de septiembre de 2017 


\section{EL CONTEXTO PESQUERO NACIONAL}

n el año 2000 se tenían identificadas en México 35,709 comunidades costeras, de las cuales $30 \%$ eran comunidades rurales con menos de 15,000 habitantes (Gabriel-Morales y Perez-Damian 2006). En la mayoría de los casos, estas comunidades rurales están geográficamente aisladas y dependen principalmente de los ambientes costeros y marinos adyacentes (figura 1). Sin embargo, algunas de estas comunidades han aprendido exitosamente a vivir en equilibrio con la naturaleza a través de la cooperación; como son los casos de las comunidades pertenecientes a la Federación Regional de Sociedades Cooperativas de la Industria Pesquera de Baja California F.C.L. (FEDECOOP) $)^{1}$ (McCay 2014). En esta región, once cooperativas han logrado este equilibrio después de más siete décadas manejando de manera sustentable sus recursos pesqueros; a través de la paciente combinación de cuatro elementos: 1) la cooperación para lograr el bien común; 2) el manejo de sus recursos mano a mano con el gobierno (comanejo); 3) la adquisición de concesiones pesqueras (derechos); y 4) su liderazgo comunitario (McCay et al., 2014; Sanchez-Bajo y Roelants 2013).

Sin embargo, muchas otras comunidades costeras tienen que enfrentar diariamente presiones externas que hacen que no logren una sustentabilidad tanto ambiental como socioeconómica y de su gobernanza. Estas presiones van desde la negligencia del gobierno [e.g. la falta de visión a largo plazo (Aburto-Oropeza et al., 2016) y la constante competencia entre las dependencias de gobierno por poder y presupuesto al no estar alineadas sus atribuciones (Mancha $e t$ al., 2018)], mercados abusivos, la competencia con otras pesquerías y su ilegalidad asociada (IMCO 2013, Cisneros-Montemayor et al., 2014), las promesas de los grandes desarrollos (e.g. turísticos, mineros) que les llevaran bienestar y justicia, y recientemente el narcotráfico (Lah y Moya 2016) y los impactos ocasionados por el cambio climático (Micheli et al., 2012).

${ }^{1}$ http://www.fedecoop.com.mx (Fecha de consulta: 1 de septiembre de 2017). 
FIGURA I. Fotografía del poblado en Isla Natividad, Baja California Sur

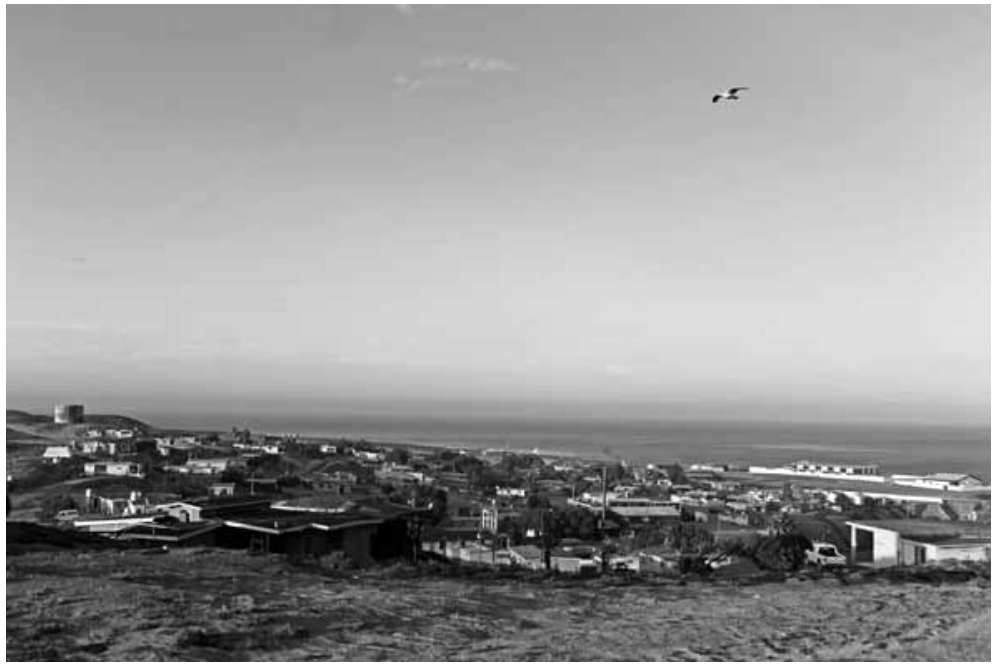

Foto: A. Hernández/coвi.

En su mayoría estas comunidades se desarrollan en un entorno pesquero de pequeńa escala o artesanal. ${ }^{2}$ En México se estima que $90 \%$ del total de pescadores $(300,000)$ son artesanales (CONAPESCA 2016), produciendo aproximadamente $40 \%$ de las capturas (Espinosa-Romero et al., 2017). Además, es importante destacar que la pesca provee trabajo indirectamente a dos millones de personas (CONAPESCA 2016); en especial a las mujeres, quienes comparten con los hombres actividades como el registro, el pago, el desembarque y la limpieza ("deschuripar") de la captura en la playa, así como en el procesamiento, venta y preparación de los productos pesque-

${ }^{2}$ Las pesquerías pueden dividirse de acuerdo al tamaño de la embarcación y capacidad de almacenamiento en dos tipos; la conocida como de pequeńa escala, ribereña o artesanal que usan embarcaciones con o sin motor fuera de borda, con una eslora (longitud) máxima total de $10.5 \mathrm{~m}$, con o sin un sistema de conservación de la captura a base de hielo y con una autonomía de tres días como máximo (Dof 2007), así como es tripulada, por lo general, entre una a tres personas (Moreno-Báez et al., 2010); y la pesca industrial o comercial se caracteriza por embarcaciones de mayor longitud y calado, con motor interior, y una mayor capacidad de almacenaje como serían las flotas para camarón altura, pelágicos menores (e.g. sardina) y el atún. 
Figura 2. Fotografía en el Mercado de la Viga en la Ciudad de México

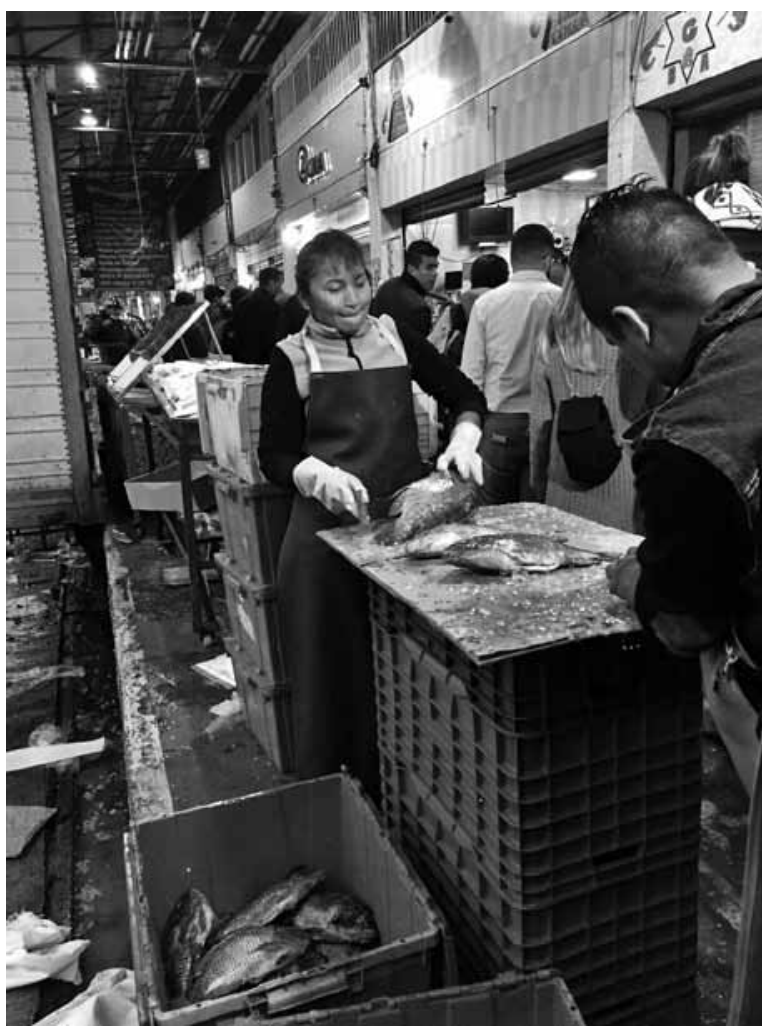

Foto: A. Lejbowicz/совI.

ros (figura 2); roles completamente invisibilizados en la mujer. Hasta la fecha no se tienen cifras sistemáticas que cuantifiquen este esfuerzo en el país; ${ }^{3}$ pero, por ejemplo, se estima que $50 \%$ de los involucrados en las pesquerías en el mundo (260 millones de personas) son mujeres (Harper et al., 2013), y la Organización de la $\mathrm{Na}$ -

${ }^{3}$ El único indicador encontrado en el último Anuario Estadístico Pesquero publicado en el 2013 por la CONAPESCA es la aprobación de motores marinos ecológicos a $321 \mathrm{mu}$ jeres de los 3,306 repartidos en total. 
ciones Unidas para la Alimentación y la Agricultura (FAO, por sus siglas en ingles) estima que en algunos países hasta $90 \%$ del procesado de pescados y mariscos es realizado por mujeres.

En el 2015, México ocupó la posición 16 de producción pesquera y acuícola en el mundo, capturando 1.75 millones de toneladas de productos pesqueros con un valor de 24 mil millones de pesos (CONAPESCA 2016). El $70 \%$ de esta producción y $60 \%$ de su valor proviene de los estados del noroeste de México (Baja California, Baja California Sur, Sonora, Sinaloa y Nayarit), ${ }_{4}^{4}$ que se caracterizan por presentar índices de marginación medios, bajos o muy bajos (Azuz-Adeath y Rivera-Arriaga 2009). Por último, es importante mencionar que de las aproximadamente 560 especies registradas en las estadísticas pesqueras nacionales, $17 \%$ están sobreexplotadas, $70 \%$ se encuentran en su máximo aprovechamiento, y $13 \%$ con potencial para desarrollarse (DOF 2012a).

\section{LAS ORgANIZACIONES DE LA SOCIEDAD CIVIL EN MÉXICO}

Las organizaciones de la sociedad civil (osc), también conocidas como organizaciones no gubernamentales (ONG), organizaciones del tercer sector o instituciones sin fines de lucro (ISFL), han ido aumentando desde los setenta con un importante crecimiento después del sismo de 1985, el cual afecto significativamente la Ciudad de México (Collin y Rafael Molina 2009; Girardo y Mochi 2012; Butcher 2014). Sin embargo, es hasta 2004 que son reconocidas legalmente, otorgándoles derechos y obligaciones, a través de la Ley Federal de Fomento a las Actividades realizadas por Organizaciones de la Sociedad Civil (Dof 2004). En el artículo quinto de esta ley se enlistan las actividades a las que se pueden dedicar las osc (cuadro 1), y en el sexto se especifican sus derechos (e.g. Art. 6, II. Participar, conforme a la Ley de Planeación y demás disposiciones jurídicas aplicables, como instancias de participación y consulta).

${ }^{4}$ Los valores se obtuvieron de la base de datos de producción 2014. (Fecha de consulta: 10 de noviembre de 2016). https://www.gob.mx/conapesca/documentos/anuarioestadistico-de-acuacultura-y-pesca 
Cuadro i. Actividades de acuerdo a la Ley Federal de Fomento a las Actividades Realizadas por Organizaciones de la Sociedad Civil (DOF 2004). En itálicas las actividades que directa e indirectamente son realizadas por las osc que promueven conservación marina y pesquerías sustentables en el noroeste de México.

I Asistencia social, conforme a lo establecido en la Ley Sobre el Sistema Nacional de Asistencia Social y en la Ley General de Salud;

II. Apoyo a la alimentación popular;

III. Cívicas, enfocadas a promover la participación ciudadana en asuntos de interés público;

IV. Asistencia jurídica;

V. Apoyo para el desarrollo de los pueblos y comunidades indigenas;

VI. Promoción de la equidad de género;

VII. Aportación de servicios para la atención a grupos sociales con discapacidad;

VIII. Cooperación para el desarrollo comunitario en el entorno urbano o rural;

IX. Apoyo en la defensa y promoción de los derechos humanos;

$\mathrm{X}$. Promoción del deporte;

XI. Promoción y aportación de servicios para la atención de la salud y cuestiones sanitarias;

XII. Apoyo en el aprovechamiento de los recursos naturales, la protección del ambiente, la flora y la fauna, la preservación y restauración del equilibrio ecológico, asi como la promoción del desarrollo sustentable a nivel regional y comunitario, de las zonas urbanas y rurales;

XIII. Promoción y fomento educativo, cultural, artístico, cientifico y tecnológico;

XIV. Fomento de acciones para mejorar la economía popular;

XV. Participación en acciones de protección civil;

XVI. Prestación de servicios de apoyo a la creación y fortalecimiento de organizaciones que realicen actividades objeto de fomento por esta ley;

XVII. Promoción y defensa de los derechos de los consumidores;

XVIII. Acciones que promuevan el fortalecimiento del tejido social y la seguridad ciudadana, $\mathrm{y}$

XIX. Las que determinen otras leyes. 
Las osc, como cualquier persona moral, deben de cumplir con obligaciones fiscales ${ }^{5}$ ante el Servicio de Administración Tributaria (sAT). Además, aquellas que están autorizadas por la Secretaría de Hacienda y Crédito Público (SHCP) para recibir donativos deducibles del impuesto sobre la renta (ISR) deben presentar un reporte anual de transparencia, ${ }^{6}$ el cual es público. En el 2012, las osc fueron consideradas como vulnerables (al igual que actividades asociadas a juegos con apuesta, concursos o sorteos, venta de autos, joyería, inmuebles, entre otros), en la Ley Federal para la Prevención e Identificación de Operaciones con Recursos de Procedencia Ilícita (Ley Antilavado) (Dof 2012b), por lo que se debe de informar con gran detalle la procedencia de los donativos ${ }^{7}$ que se obtienen.

En general se pueden distinguir cuatro características que distinguen a las osc de las organizaciones o instituciones del Estado y el mercado: 1) actúan en nombre del bien público; 2) no buscan un fin lucrativo o de ganancia monetaria, sino de ganancias de valores de uso no monetario y bienes relacionados a intangibles (e.g. el bien común, justicia social, equidad de género, ambiente sano, entre otros); 3) los recursos que usan, humanos y financieros, provienen de donaciones y voluntariado; y 4 ) están formadas por personas que creen en los ideales y motivaciones de las organizaciones y no por obtener una ganancia monetaria (Girardo y Mochi 2012). Las OsC, a diferencia de otras organizaciones, tienen características (BermejoJiménez 2007) que las hacen más competitivas, como su flexibilidad, empatía y compromiso, credibilidad y confianza, aproximación holística a la problemática social y ambiental, destrezas y especialidades, redes sociales, desarrollo de liderazgo, y recursos.

El Instituto Nacional de Estadística y Geografía (INEGI) reporta que para el 2015, $43 \%$ de los 3.3 millones de personas que trabajan

${ }^{5}$ Personas morales con fines no lucrativos. http://www.sat.gob.mx/informacion_fiscal/obligaciones_fiscales/personas_morales/no_lucrativas/Paginas/otras_obligaciones.aspx (Fecha de consulta: 10 de noviembre de 2016).

${ }^{6}$ Artículo 82, fracción VI, de la Ley del Impuesto sobre la Renta; regla I.3.9.10, artículo décimo primero transitorio de la Resolución Miscelánea Fiscal para 2014 y su ficha 20/ISR: "Información para garantizar la transparencia, así como el uso y destino de los donativos recibidos" del anexo 1-A.

${ }^{7}$ Artículo 17, fracción XIII. 
en las osc eran remuneradas, y el resto eran voluntarios. Así como su aportación a la economía nacional era de 3.1\% (\$531,404,000 pesos) con respecto al Producto Interno Bruto (PIB). ${ }^{8}$

Con información más reciente, en julio de 2017, el Registro Federal de Organizaciones de la Sociedad Civil tenía registrados en el país 36,731 actores sociales de los cuales, en mayo del mismo año, solamente 8,832 contaban con la autorización para recibir donativos deducibles del ISR. ${ }^{9}$ Por último, se estima que solamente $2.8 \%$ de las osc están enfocadas a cuestiones ecológicas y especies en protección y peligro, y $2.1 \%$ a la investigación científica y tecnología; la gran mayoría (90\%) están en los rubros de asistencia social, educación y cultura.

En el noroeste de México, las osc denominadas como ambientalistas (aquellas que buscan el bien ambiental, que es un bien público) surgieron a mediados de los ochenta, las cuales fueron en su mayoría osc internacionales (i.e. Conservation International, CI; The Nature Conservancy, TNC; World Wildife Fund, wwF) que hacían alianzas con las nuevas y escasas ocs mexicanas (Herrmann 2004), así como abrían sus propios programas regionales (e.g. Golfo de California). Estas organizaciones, así como otras en el mundo, impulsaron la visión de conservar las especies en peligro y ecosistemas amenazados usando las áreas naturales protegidas (ANP) como principal herramienta, y trabajando con los gobiernos y la academia, no necesariamente con las comunidades costeras directamente impactadas (positiva o negativamente) (Sánchez-Ibarra et al., 2013, Luque-Agraz y Gómez 2007).

Un ejemplo emblemático de esta tendencia en los noventa fue el establecimiento de la Reserva de la Biósfera Alto Golfo de California y Delta del Río Colorado (RBAG), la cual fue decretada en 1993

\footnotetext{
${ }^{8}$ http://www.inegi.org.mx/est/contenidos/proyectos/cn/isfl/ (Fecha de consulta: 1 de septiembre de 2017). Este valor es 15 veces mayor que lo que aporta la pesca y la acuacultura al PIB. Por ejemplo, en el 2016 el valor de la captura pesquera fue de 35,000 millones de pesos. (http://www.sagarpa.gob.mx/Delegaciones/distritofederal/boletines/ Paginas/JAC_00206_11.aspx (Fecha de consulta: 9 de septiembre de 2017).

${ }^{9}$ https://www.cemefi.org/programas/informacion-3er-sector.html (Fecha de consulta: 1 de septiembre de 2017).
} 
(Cisneros-Montemayor y Vincent 2016). En aquellos años existía fuerte discrepancia en opiniones entre expertos de las ciencias sociales y biológicas conforme al establecimiento de esta ANP; por ejemplo, la opinión de reconocidos antropólogos sobre la RBAG era: "Francamente, nos oponemos a lo que percibíamos como un complejo imperialismo medioambiental, el cabildeo político de la organización internacional de cetáceos al servicio del libre comercio y la agenda neoliberal del presidente Salinas" 10 (McGuire y Ciria Valdez-Gardea 1997), mientras ONG ambientalistas lo veían como un éxito. ${ }^{11}$ Aún faltarían dos décadas para comenzar a escuchar el uso y aplicación de modelos socioecológicos (Leslie et al., 2015), manejo ecosistémico (e.g. Munguia-Vega et al., 2015), y ciencia ciudadana (López Sagástegui et al., 2014, Fulton et al., en prensa).

Sin embargo, es importante destacar, que paralelamente al proceso de RBAG, se decretaron otras ANP en el Golfo de California por iniciativas directamente de las comunidades locales, y no de las osc. Estas comunidades no deseaban tener en sus áreas de trabajo de pesca artesanal y de turismo a la pesca industrial (i.e. barcos de arrastre de camarón y pesca de pelágicos menores como la sardina), como fueron los casos de los parques nacionales Cabo Pulmo (decretado en 1995) (CONANP 2006 ${ }^{12}$ y Bahía de Loreto (decretado en 1996) (CONANP 2000).

A principios del 2000, comenzaron a aumentar el número de osc mexicanas con objetivos ambientales costeros y marinos, incorporando la visión y necesidades de las comunidades costeras en sus

10 "We frankly objected to what we perceived as a complex environmental imperialism -the marshalling of the international cetacean lobby in the service of President Salinas's free trade and neo-liberal agenda" (McGuire y Ciria Valdez-Gardea 1997).

${ }^{11}$ Observación del primer autor al proceso de declaratoria de la RBAG, quien participó en algunas reuniones como asistente durante el diseńo de la RBAG; recuerda que los participantes no estaban en contra de las comunidades, sino que existía una verdadera ignorancia de cómo integrar la parte socioeconómica y ambiental de forma sistemática y adecuada, aunada a posiciones no reconciliables entre los "biólogos" y los "sociólogos". No existía voluntad de ambos gremios, científicos biológicos y sociólogos, en colaborar y construir. El resultado de esta falta de colaboración se puede revisar en Vince (2017).

${ }^{12}$ Actualmente el Parque Nacional Cabo Pulmo es un ejemplo a nivel mundial de recuperación del ecosistema y el empoderamiento comunitario. http://octavioaburto. com/cabo-pulmo (Fecha de consulta: 9 de septiembre de 2017). 
acciones. Así como impulsando el comanejo entre las comunidades y el gobierno (Basurto et al., 2000), el uso de ecocertificaciones para productos pesqueros (Smith et al., 2010), el uso del conocimiento local para diseñar reservas marinas completamente protegidas (Cudney-Bueno et al., 2009), y el monitoreo comunitario (ciencia ciudadana) (López Sagástegui et al., 2014, Fulton et al., en prensa).

Actualmente el rol de algunas osc consideradas "ambientalistas" en el noroeste de México ha pasado de un enfoque únicamente ambiental a promover pesca sustentable integrando a todos los actores a través de apoyar: 1) reuniones (planeación, logística, facilitación, mediación); 2) la producción, análisis y comunicación de información; y 3) la elaboración y el seguimiento de acuerdos y reglas, y de asegurar la representación, empoderamiento y capacitación de los distintos sectores para llegar a decisiones incluyentes, participativas y consensuadas (Espinosa-Romero et al., 2014a). En un reciente taller de trabajo (noviembre 2016), entre más de 10 ocs del noroeste de México, todas manifestaron la importancia de trabajar desde las comunidades para lograr objetivos de conservación antes de impulsar decisiones sin incluir su participación.

\section{UNA OSC}

Comunidad y Biodiversidad, A.C. (COBI) ${ }^{13}$ comenzó en 1999 en el cuarto de lavadora de uno de los socios fundadores durante sus estudios doctorales en Bahía de Kino (Sonora) junto con otros cuatro amigos. Este grupo de jóvenes veía como la diversidad y abundancia de la vida marina y la calidad de vida de las comunidades costeras en México estaban disminuyendo drásticamente. Después de casi dos décadas, COBI continúa trabajando en poder lograr que las comunidades costeras de México sean corresponsables del cuidado de los mares mediante la promoción de la participación efectiva de los sectores involucrados en proyectos de conservación y pesca sustentable. СОвi ha trabajado con una pequeña, pero heterogénea muestra de estas comunidades costeras en tres ecorregiones prioritarias

\footnotetext{
${ }^{13}$ www.cobi.org. $\mathrm{mx}$
} 
para la conservación marina en México: 1) la Península de Baja California en el Océano Pacífico; 2) la Región de las Grandes Islas en el Golfo de California; y 3) el Arrecife Mesoamericano en el Mar Caribe. Сов realiza su trabajo a través de cuatro líneas estratégicas: 1) el fortalecimiento de líderes y organizaciones pesqueras; 2) la promoción de estándares internacionales de pesca sustentable; 3) el uso de reservas marinas completamente protegidas para restaurar ecosistemas marinos; y 4) comunicar los casos de éxito para mejorar la política pública.

La participación de las comunidades costeras es un ingrediente clave en las intervenciones de COBI; esta participación está definida como el proceso en que la comunidad (i.e. los actores que tienen una relación directa con una pesquería o buscan su aprovechamiento sustentable) está involucrada de manera equitativa y justa en la toma de decisiones y la implementación efectiva de las mismas. Un proceso equitativo y justo significa buscar la inclusión de los grupos tradicionalmente no representados en la toma de decisiones, desde el diagnóstico hasta la propuesta de soluciones, implementación y evaluación, tomando en cuenta la ciencia no tradicional, un balance de actores en la mesa de negociación y una evaluación externa al gobierno de los alcances de la solución para que prevalezca el bien común (e.g. Ostrom 2000).

\section{ACción Sin Daño}

Para el presente análisis se utilizó el marco que proporciona la metodología Acción Sin Daño (Wallace 2014), la cual fue desarrollada en los noventa para evaluar el impacto de los proyectos humanitarios y de paz, y que aquí se ha adaptado para medir el impacto de una OsC enfocada a la conservación marinas y manejo sustentable pesquero. Ésta es una poderosa metodología de reflexión que mide más allá de las tradicionales métricas [resultados (outcomes) y productos (outputs)] requeridas por los financiadores y el gobierno, y que confronta constantemente la ética y las acciones que se realizan en un proyecto en donde las osc son agentes externos en una comunidad (e.g. Birdsall 2007). 
El marco metodológico está basado en analizar el contexto de la intervención (¿qué?, ¿por qué?, ¿para qué?, ¿cuándo?, ¿dónde?, ¿cómo?, ¿quiénes?, ¿con quiénes?), qué actividades, instituciones, personas, actitudes, intereses, valores, necesidades, experiencias y símbolos potencializan las conexiones (conectores) y cuáles promueven las divisiones (divisores) (Wallace 2014, ccc 2016). En este trabajo se analizan los conflictos producidos por СОвı al hacer la transferencia de recursos (i.e. efectos de distribución, sustitución, legitimación y mercado), así como la producción de mensajes éticos implícitos (i.e. valores diferentes para vidas diferentes, publicidad que polariza, tensiones y sospechas, armas y poder, competencia entre organizaciones/empresas, e impunidad) (ccc 2016).

\section{Contexto en CADA Sitio}

En esta sección se presenta un análisis comparativo de cinco proyectos realizados por COBI que promueven el establecimiento de reservas marinas completamente protegidas (zonas de no pesca, restauración, recuperación, refugios, entre otras designaciones), ${ }^{14}$ como una herramienta de recuperación de especies, pesquerías y ecosistemas, en pequeñas comunidades pesqueras (menos de 5,000 habitantes). Las comunidades dentro del Golfo de California fueron Bahía de Kino y Puerto Libertad en Sonora y Ligüí en Baja California Sur, y en este último estado, pero en el lado del océano Pacífico, en las islas Natividad y Magdalena. A continuación, se hace una breve descripción del contexto para cada uno de los sitios, y en el cuadro 2 se presenta una comparación entre los sitios en términos de conectores, divisores, transferencia de recursos y mensajes éticos implícitos que impactaron negativamente la intervención.

\footnotetext{
${ }^{14}$ Las designaciones legales de las zonas de no pesca pueden ser como Zona Núcleo dentro de una ANP administradas por la CONANP en la Secretaría de Medio Ambiente y Recursos Naturales (SEMARNAT) y zonas de refugio por la CONAPESCA en la Secretaría de Agricultura, Ganadería, Desarrollo Rural, Pesca y Alimentación (SAGARPA) (Torre et al., 2016). Además, existen las zonas designadas voluntariamente por acuerdo comunitarios y aquellas que por seguridad nacional (e.g. plataformas petroleras y termoeléctricas) está prohibido la extracción pesquera (Torre et al., 2016).
} 


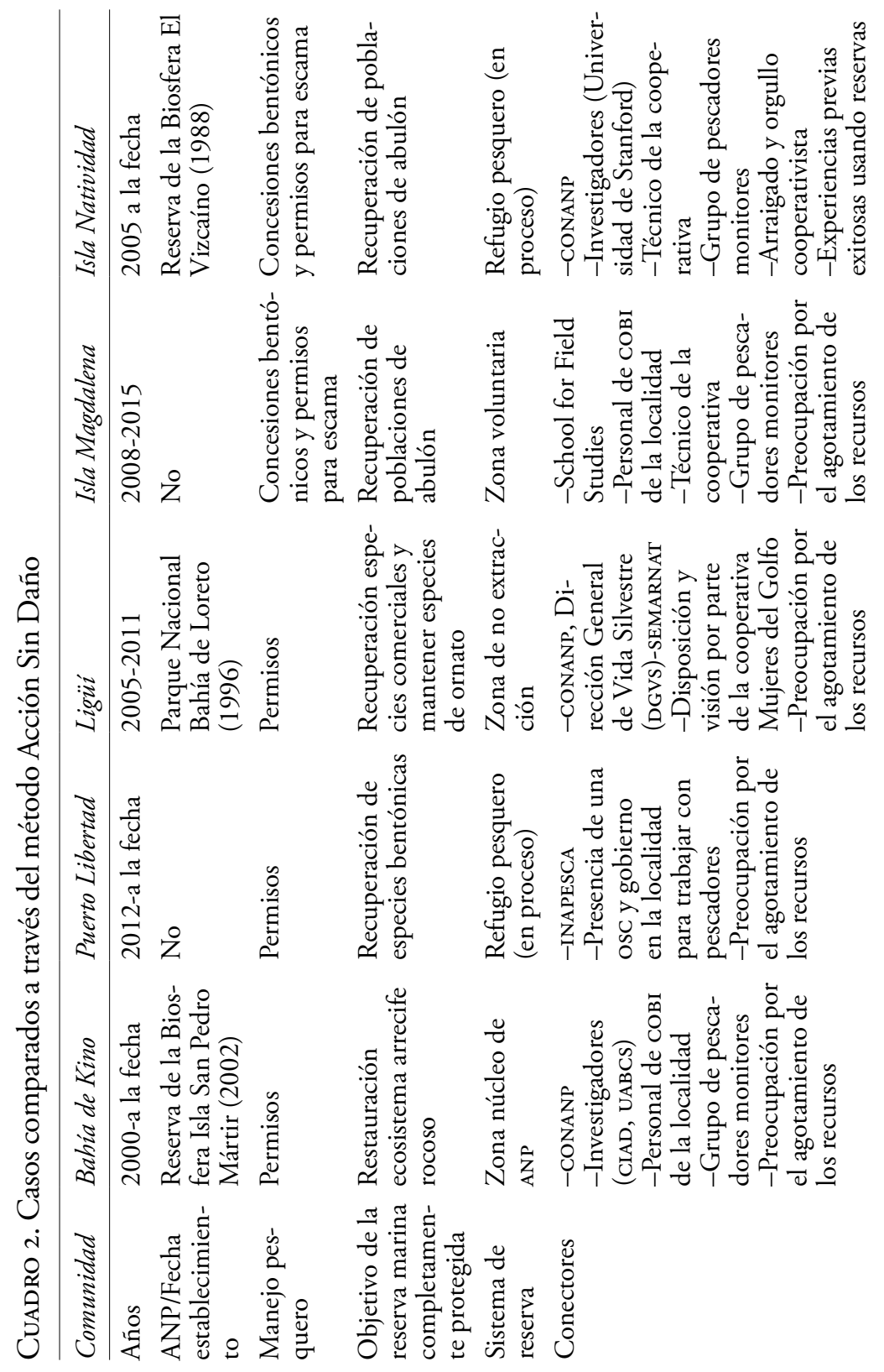




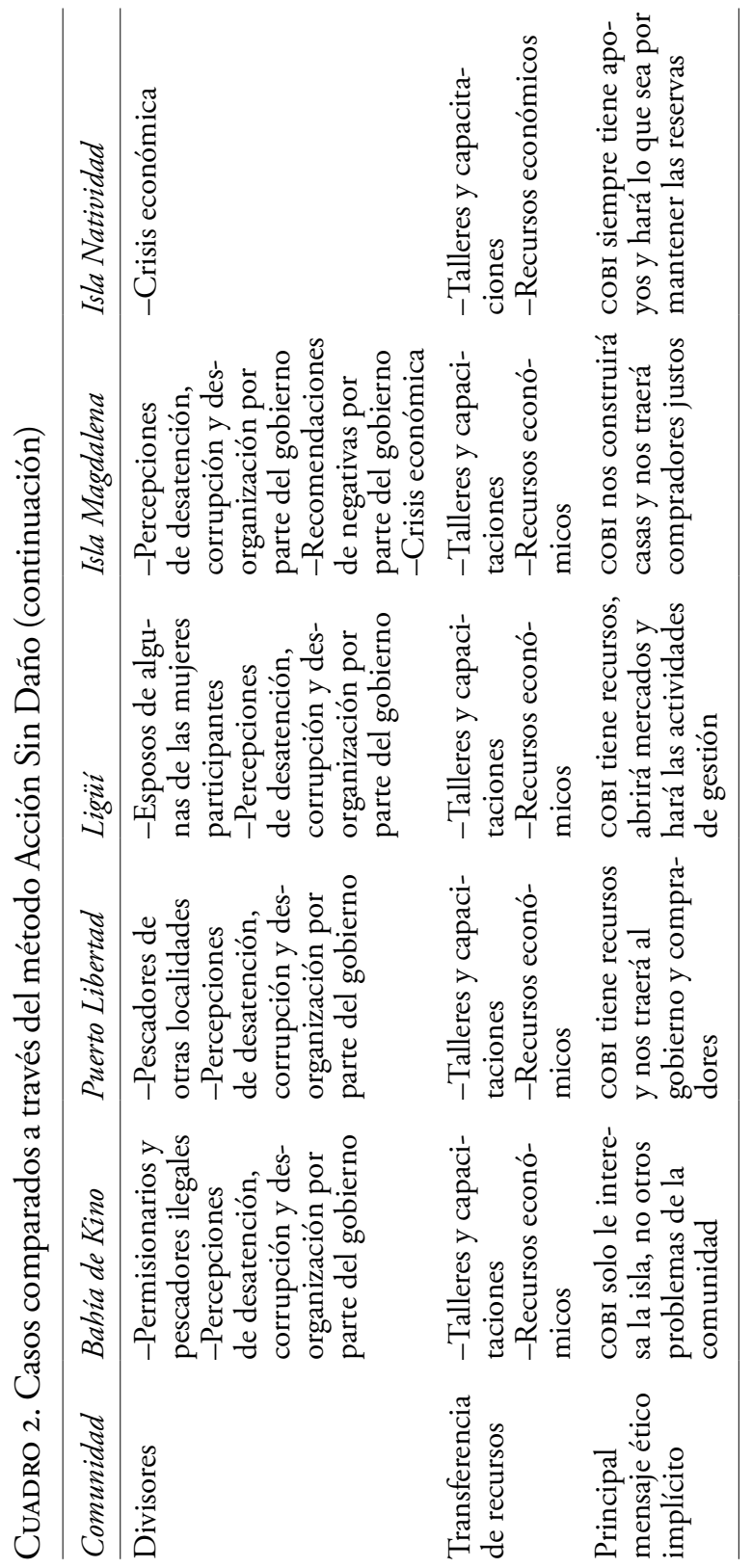


1. Isla San Pedro Mártir es la isla más oceánica en el Golfo de California, ubicada a $60 \mathrm{~km}$ de cada costa, sin asentamientos permanentes. ${ }^{15}$ En el 2002, la isla ${ }^{16}$ junto con sus aguas adyacentes fueron decretadas con la categoría de Reserva de la Biosfera, y en el 2011 se publicó su plan de manejo. Las aguas alrededor de la isla son usadas principalmente por buzos comerciales (Meza et al., 2009) y por pescadores deportivos de Estados Unidos (Fujitani et al., 2012), ambos grupos provenientes de Bahía de Kino, un poblado de menos de 5,000 habitantes a $100 \mathrm{~km}$ de la capital del estado, Hermosillo. La isla es conocida por los pescadores ribereños como "el guardadito", ya que siempre hay producto en la isla, y si se tuvo una mala pesca, se puede pasar por algo a la isla (Cudney-Bueno et al., 2009, Torre y Rojo 2011). СОві ha estado involucrado desde el diseño del ANP en el 2000, y en distintos grados (i.e. como principal promotor, colaborador, apoyo, o seguimiento) a lo largo de casi dos décadas en acciones asociadas a investigación, conservación marina y promoción de pesca sustentable. El diseño de la zona núcleo de esta ANP fue a través de talleres con los buzos comerciales (CudneyBueno et al., 2009), y desde el 2007 se comenzó un programa de capacitación a ocho buzos comerciales para que ellos realizaran el monitoreo submarino con la finalidad de que pudieran evaluar los efectos de no pescar en la salud de las poblaciones comerciales y el ecosistema en un ANP. Este grupo de buzos ha formado su propia compañía, Grupo de Monitoreo Submarino y Análisis de Cambio Climático S.C. de R.L. de C.V. (Torre y Rojo 2011), y son quienes ahora realizan el monitoreo submarino apoyado a través de subsidios que aporta la Comisión Nacional de Areas Naturales Protegidas (CONANP).

2. Puerto Libertad es una comunidad costera de menos de 3,000 personas ubicada a $189 \mathrm{~km}$ al norte de Bahía de Kino. Las dos prin-

${ }^{15}$ La isla fue habitada por esclavos de la comunidad yaqui en los ańos 1850 durante la explotación del guano (Bowen 2004); así como es usada en pequeńas temporadas (semanas) por investigadores (en los noventa) y pescadores artesanales.

${ }^{16}$ La parte terrestre de las más de 900 islas en el Golfo de California están resguardadas a través del Área de Protección de Flora y Fauna-Islas del Golfo de California, y a finales de los noventa se comenzó un proceso de incluir las aguas adyacentes de algunas de las islas bajo protección, siendo uno de los casos la Isla San Pedro Mártir. 
cipales actividades son la pesca artesanal y el empleo que provee la termoeléctrica. Esta comunidad es reconocida por ser la que viaja más lejos para pescar en el Golfo de California Norte (Moreno-Báez et al., 2012), se podrían considerar como los pescadores ribereños "oceánicos" de la región. En el 2012, coBi obtuvo apoyo de la Red Nacional de Información e Investigación en Pesca y Acuacultura (RNIIPA) del Instituto Nacional de la Pesca (INAPESCA) para implementar el enfoque ecosistémico al manejo de pesquerías ribereñas en esta localidad (Espinosa-Romero et al., 2014b). En la primera fase del proyecto se implementaron tres pasos, siempre de la mano con los pescadores de la comunidad: 1) la caracterización de los ecosistemas marinos a través del conocimiento local;2) la definición de los objetivos e indicadores para implementar el manejo ecosistémico; y 3) el diseño e implementación de una propuesta de manejo consensuada entre los principales usuarios (pescadores libres, así como para cooperativas y permisionarios) de los recursos pesqueros de esta localidad, incluyendo en la propuesta una zona de refugio pesquero para la recuperación de especies bentónicas (almejas, callos y pulpos) y peces comerciales. La zona de refugio fue decretada en julio del 2017, después de un proceso de dos años por parte del gobierno (DOF 2017). Al igual que en Bahía de Kino, en esta comunidad también se capacitaron buzos para el monitoreo submarino, y para que ellos puedan realizar la evaluación de sus recursos (Fulton et al., en prensa).

3. Ligüi es un pequeño poblado (menos de 200 personas) costero a $37 \mathrm{~km}$ al sur de la ciudad de Loreto dentro del Parque Nacional Bahía de Loreto. La principal actividad es la pesca artesanal de especies bentónicas, a través del buceo, y especies de peces (escama) con redes y anzuelo. El proyecto que se realizó en esta comunidad fue asociado con la explotación sustentable de especies de ornato (invertebrados y peces) por parte de la Sociedad Cooperativa de Producción Pesquera (SCPP) Mujeres del Golfo usando una combinación de herramientas de manejo pesquero, cuotas de captura y zonas de no extracción (SEMARNAT 2012; Germain et al., 2015). El proyecto comenzó en el 2005, finalizó la intervención en el 2011. A lo largo del proyecto se capacitó al grupo de ocho mujeres que formaban la 
cooperativa en el manejo sustentable de las especies de ornato, su manejo, transportación y venta de las especies capturadas, así como en el monitoreo submarino y la generación de información para el manejo de estas especies.

4. Isla Magdalena es la más grande del complejo lagunar Bahía Magdalena. En la isla está la scpp Bahía Magdalena, que tiene los permisos para la explotación de escama y concesiones de recursos bentónicos (abulón y langosta). También en la isla se realizan actividades de turismo, como la pesca deportiva y la observación de ballenas. En ella habitan 200 personas, pero puede aumentar a 500 personas dependiendo la pesquería que se esté realizando en la temporada. Сов I trabajó con la cooperativa entre el 2008 y 2015 en el diseño, implementación y evaluación de una reserva marina voluntaria (Villaseñor-Derbez et al., 2015). Como parte del proceso, se hicieron talleres, así como se capacitó a los buzos comerciales de la comunidad en técnicas de monitoreo submarino (Fulton et al., en prensa). En el 2009, las casas de la comunidad quedaron en su mayoría destruidas por el huracán Jimena, en ese mismo año se había establecido formalmente entre СОВI y la cooperativa, la reserva voluntaria. La comunidad no podía permitirse dejar de pescar ante tal situación, por lo que COBI se comprometió a buscar soluciones para reconstruir el pueblo a cambio de que no se abriera la reserva. Entre 2009 y 2014 se trabajo con miembros de esta comunidad en el diseño y construcción de la Asamblea de Socios de la cooperativa, usando ecotecnologías (Murillo y Lejbowicz 2014); así como en planes para continuar la construcción de viviendas. Sin embargo, en enero 2015, la cooperativa decidió abrir la reserva debido a una crisis económica interna; por lo que solicitaron mayor cuota de captura de abulón (Haliotis spp) a investigadores del InAPESCA (Instituto Nacional de Pesca y Acuacultura), quienes sugirieron abrir la reserva para obtener mayor cuota (P. Sierra, comn. pers.). En esta misma fecha la cooperativa dio aviso a COBI de no querer continuar colaborando con la organización.

5. Isla Natividad es una pequeña isla (7 km de largo) a $8 \mathrm{~km}$ de la costa, donde habitan los socios de scpp Buzos y Pescadores de la Baja California y los miembros de sus familias, con un total de 
aproximadamente 400 personas. La cooperativa pesca principalmente especies bentónicas (abulón, langosta, caracol y pepino de mar) en su concesión pesquera, así como especies de escama con cańa y anzuelo y redes. La cooperativa es miembro de las 11 cooperativas que forman la Federación Regional de Sociedades Cooperativas de la Industria Pesquera Baja California (FEDECOOP), que son reconocidas a nivel mundial por el manejo pesquero sustentable que realizan (McCay et al., 2014). Como ejemplo, la langosta que producen está certificada como pesquería sustentable por el Marine Stewardship Council (MCS) (Smith et al., 2010). coBi y la cooperativa comenzaron a colaborar en el 2005, estableciendo una reserva voluntaria desde el 2006. El rol de COBI en la isla ha sido, junto con investigadores principalmente de la Universidad de Stanford, impulsar las inquietudes provenientes de los pescadores alrededor del manejo y conservación de los recursos marinos en la isla. Por ejemplo, en el 2015 se desarrollaron talleres para identificar intereses de los pescadores, reconociéndose cuatro temas: 1) los efectos y costos de las reservas marinas; 2) la transferencia del conocimiento local dentro de la comunidad y mas allá; 3) desarrollo de alternativas pesqueras y acuícolas; y 4) los monitoreos comunitarios. Al tener claro estos intereses se desarrollaron y se están implementando estrategias de trabajo en conjunto.

\section{ANÁLISIS}

Conectores. En los cinco casos se identificaron como conectores clave las personas que eran originarias de la localidad y que laboraban en COBI o los técnicos que trabajan para las cooperativas pesqueras. El papel de ellos en todos los casos fue de desarrollar y mantener una constante comunicación con los involucrados de las comunidades en el proyecto sobre los objetivos y alcances del mismo proyecto. Una de sus actividades más comunes fue desenmarañar los malos entendidos, rumores y chismes creados por los divisores (e.g. percepciones preconcebidas); la transferencia de recursos (e.g. contratación de alimentos y hospedaje a determinados miembros de la comunidad); y por los mensajes implícitos (e.g. COBI tiene recursos 
suficientes y hará lo que sea por mantener las reservas) creados a lo largo del proyecto. Otro importante conector fue la conanp en los casos que el trabajo se realizó dentro de una ANP, y en el caso de Puerto Libertad, el papel que jugó por su presencia y liderazgo el INAPESCA, fue esencial para reforzar ante la comunidad el compromiso de la osc en el proyecto. En todos los casos, fueron conectores esenciales los investigadores involucrados en las intervenciones a través de la validación e incorporación del conocimiento local en el diseño, implementación y monitoreo de las reservas marinas, así como en la cocreación de nuevo conocimiento entre los pescadores y los investigadores. Por último, un conector fue la preocupación generalizada por el agotamiento de los recursos pesqueros y la justa aplicación de la ley.

Divisores. El principal divisor en todos los casos fueron las percepciones existentes. Por una parte los habitantes de las comunidades expresan su frustración y desconfianza a proyectos por la desatención, corrupción, desorganización y falta de inspección y vigilancia por parte de las agencias de gobierno, y, por otra, el que las ocs sólo quieren prohibir el desarrollo y promueven proyectos de intereses extranjeros. Otro divisor fue la burocracia, en especial, los tiempos de respuesta a trámites para obtener, por ejemplo, permisos de pesca o lograr decretos de normas, ordenamientos, planes de manejo y refugios pesqueros (INAPESCA y CONAPESCA), y planes de manejo por parte de la CONANP. Estos largos tiempos de espera, de hasta años, ${ }^{17}$ ocasionaron en repetidas veces, alimentar el ciclo de las percepciones negativas preexistentes. Otro divisor fue colaborar con ciertas personas de la comunidad, que produjeron un sentimiento negativo, y aumentaron la desconfianza por parte de socios de las cooperativas y de los pescadores libres. Un caso particular se presentó en el proyecto en la comunidad de Ligüi, donde algunos de los esposos no apoyaron el proyecto liderado por una cooperativa de mujeres.

Transferencia de recursos. En todos los proyectos se realizaron talleres y capacitaciones a los pescadores. Así como se transfirieron

${ }^{17}$ Por ejemplo, el Plan de Manejo de Parque Nacional Bahía de Loreto no se ha publicado desde su revisión en el 2009; o permisos de pesca que tardaron hasta dos años, o simplemente nunca llegaron. 
recursos económicos a miembros de las comunidades asociados a hospedaje y alimentación, ésta se procuró hacer de forma homogénea, pero en lugares pequeños (las islas) los alimentos se consumieron en lugares específicos (por disposición, salubridad y servicio), que ocasionaron tensiones en las comunidades. También se pagaron salarios a las personas que colaboran en los monitoreos, y la renta de embarcaciones y gasolina asociada, con excepción del caso de Isla Natividad, donde la cooperativa cubrió estos últimos gastos. Los participantes en las actividades de monitoreo fueron seleccionados por convocatoria abierta (Bahía de Kino, Puerto Libertad) o por las cooperativas (Ligüí, y las islas Magdalena y Natividad). Por otra parte, en todos los casos, el rol de coBI como gestor en trámites con el gobierno ocasionó una fuerte dependencia de los usuarios. En el caso de Isla Magdalena incluso se llegó a efectos de sustitución en donde se asumieron responsabilidades que no le correspondían a la OsC.

Mensajes éticos implícitos. Se identificaron dos grandes mensajes implícitos que ocasionaron confusión y molestia en las comunidades. El primero fue que a COBI únicamente le interesaban las reservas marinas y no la situación de la comunidad, promoviendo una división en la comunidad entre los que están a favor o en contra de lo que hace la osc. En algunos casos se hablaba de "las reservas de COBI". El otro mensaje fue que COBI haría lo que fuera necesario por mantener las reservas. Estos mensajes ocasionaron en más de una ocasión que los divisores encontrados se potencializaran negativamente.

\section{Conclusiones}

Aún falta por hacer y demostrar de parte de las osc ambientalistas para cambiar la percepción que se tiene de ellas; de entes que cumplen los objetivos de quienes aportan los apoyos financieros (extranjeros) a organizaciones mexicanas preocupadas y enfocadas en lograr el bienestar social y ambiental del país, promoviendo la colaboración, la participación, la inclusión y la transparencia. Sin embargo, para lograrlo las osc aún necesitan lograr una visión compartida entre ellas mismas y las comunidades (e.g. pescadores, investigadores) con las que colaboran; así como procedimientos y roles claros 
por parte de las osc que aseguren una colaboración y participación efectiva (Espinosa-Romero et al., 2014a). Por ejemplo, una práctica que se comenzó a usar fue presentar la visión de COBI, tanto en términos operativos como financieros. Así como ser claros, en el caso de las reservas voluntarias, que la decisión final siempre sería de las cooperativas.

En este breve análisis se ha identificado un factor determinante que pasa de un conector que promueve adecuadamente un proyecto a convertirse en un divisor, ésta es "la pasión" de las personas que diseñan e implementan la iniciativa desde la osc. Cuando se deja de escuchar a las comunidades pesqueras y se entra a una actitud paternalista (como la que se le critica al gobierno), muy probablemente los proyectos, a pesar de ser nobles, serán un fracaso tarde o temprano. Otro importante divisor es la eterna lucha de poder disfrazada por sus ámbitos de acción y protegida por la ley entre el sector de gobierno a cargo de la administración e investigación pesquera y las áreas naturales protegidas, que puede provocar una parálisis total y caída de cualquier iniciativa.

Además de estos divisores, se identificaron importantes conectores: como el conocimiento local sobre los recursos marinos; la acción colectiva por el bien común; y la identificación de distintos liderazgos en las comunidades. Estos conectores son claves ya que generan confianza en la comunidad y hacen más transparente el tipo de intervención que se quiere realizar para la implementación de proyectos en comunidades costeras. Las comunidades se sienten partícipes en la resolución de las problemáticas y en el manejo de sus recursos.

La metodología acción sin daño es una herramienta que debe de implementarse para realizar las reflexiones de las intervenciones que hacen los agentes externos a una comunidad a lo largo de un proyecto. Las ocs deben de asumir los impactos (positivos y negativos) que hacen en pequeñas comunidades rurales, donde las necesidades y problemáticas locales (e.g. económicas, sociales y de gobernanza) son más una prioridad que los objetivos de conservación y sustentabilidad (agenda de la osc) propuesta en ese momento. En estas ocasiones es importante realizar un diagnóstico exhaustivo, el cual 
permitirá fortalecer a la comunidad y a sus líderes natos en temas de su interés. Además de buscar el bien común social y ambiental de su comunidad integrando otros actores.

\section{BiBLIOGRAFÍA}

Aburto-Oropeza, Octavio, Catalina López-Sagástegui, Marcia Moreno-BÁez, Ismael Mascareñas-Osorio, Víctor JimÉnezEsquivel, Andrew F. Johnson y Brad Erisman. 2017. "Endangered Species, Ecosystem Integrity, and Human Livelihoods". Conservation Letters (2017): 9. https://doi.org/10.1111/conl. 12358

Azuz-Adeath, Isaac y Evelia Rivera-Arriaga. 2009. “Descripción de la dinámica poblacional en la zona costera mexicana durante el periodo 2000-2005". Papeles de Población 15(62) (octubrediciembre 2009): 75-107.

Basurto, Xavier, Luis Bourillon y Jorge Torre. 2000. "The Role of a Non-Governmental Organization (NGO) In An Emerging Co-Management Regime: The Mexican Seri Indians' Case Study". The 8th Biennial Conference of the International Association, Bloomington, Indiana, 30 mayo- 4 junio, 2000.

BERMEJO-JimÉnEZ, José Ernesto. 2007. "Las organizaciones de la sociedad civil en México". Bien Común 13(151): 25-28.

Birdsall, Nancy. 2007. "Do No Harm: Aid, Weak Institutions and the Missing Middle in Africa". Development Policy Review 25(5): 575-598.

Bowen, Thomas. 2000. Unkown Island. Seri Indians, Europeans, and San Esteban Island in the Gulf of California. Alburqueque: University of New Mexico Press.

Butcher, Jacqueline. "Tercer sector y desarrollo en México." En Del "vivir bien" al "buen vivir" entre la economía feminista, la filantropia y la migración: hacia la búsqueda de alternativas, coord. Alicia Girón, 91-111. Colección de Libros Problemas del Desarrollo, México: unAm Revista Latinoamericana de Economía, 2014.

Centro de Colaboracón Civica (cCC). 2016. Taller Acción Sin 
Daño 2016. https://prezi.com/wffd5elnzu-z/taller-accion-sindano-viento-fuerte 2016/?utm_campaign=share\&utm _ medium=copy (Fecha de consulta: 10 de noviembre de 2016 Cisneros-Montemayor, Andrés, Miguel Angel Cisneros-Mata, Sarah Harper y Daniel Pauly. 20I 3 . "Extent and Implications of IUU Catch in Mexico's marine fisheries". Marine Policy (39): 283-288.

Cisneros-Montemayor, Andres y Amanda Vincent. 2016. "Science, Society, and Flagship Species: Social and Political History as Keys to Conservation Outcomes in the Gulf of California”. Ecology and Society 21(2): 9.

Collin, Laura y Rafael Molina. 2009. "Las Organizaciones de la Sociedad Civil en México: de la invisibilidad al protagonismo". Colloques DOI:10.4000/nuevomundo.47723

Comisión Nacional de Áreas Naturales Protegidas (coNANP). 2000. Plan de Manejo del Parque Nacional Bahía de Loreto. México: Semarnar.

. Plan de Manejo del Parque Nacional Cabo Pulmo. 2006. México: SEMARNAR.

Comisión Nacional de Acuacultura y Pesca. 2016. "Sube México en el ranking de producción pesquera internacional de la FAO". http://www.sagarpa.gob.mx/Delegaciones/nayarit/boletines/Paginas/B0512016.aspx (Fecha de consulta: 10 de noviembre de 2016).

Cudney-Bueno, Richard, Luis Bourillón, María Andrea SáenzArroyo, Jorge Torre, Peggy Turk-Boyer y William Shaw. 2009. "Governance and Effects of Marine Reserves in the Gulf of California, Mexico". Oceans \& Coastal Managament 52(3-4): 207-218.

Diario Oficial de Federación (DOF). 2004. "Ley Federal de Fomento a las Actividades Realizadas por Organizaciones de la Sociedad Civil". México: Secretaría de Gobernación.

2007. "Ley General de Pesca y Acuacultura Sustentables". México: Secretaría de Gobernación.

2012a. "Carta Nacional Pesquera". México: Secretaría de Gobernación. 
. 2012b. "Ley Federal para la Prevención e Identificación de Operaciones con Recursos de Procedencia Ilícita”. México: Secretaría de Gobernación.

. 2017. "Acuerdo por el que se establece una Zona de Refugio Pesquero Total Temporal en aguas marinas de jurisdicción federal de Puerto Libertad, Municipio de Pitiquito en el Estado de Sonora”. México: Secretaría de Gobernación.

Espinosa-Romero, María José, Laura F. Rodríguez, Amy Hudson Weaver, Cristina Villanueva-Aznar y Jorge Torre. 2014a. "The Changing Role of NGOs in Mexican Small-Scale Fisheries: From Environmental Conservation to Multi-Scale Governance". Marine Policy, vol. 50: 290-299.

Espinosa-Romero, María José, Miguel Angel Cisneros-Mata, Timothy McDaniels y Jorge Torre. 2014b. "Aplicación del enfoque ecosistémico al manejo de pesquerías artesanales. Caso de estudio: Puerto Libertad, Sonora”. Ciencia Pesquera 22(2): 65-77. Espinosa-Romero, María José, Jorge Torre, José Alberto ZepedA, Francisco Vergara-Solana y Stuart Fulton. 2017. "Civil Society Contributions to the Implementation of the Voluntary Guidelines for Securing Sustainable Small-Scale Fisheries in Mexico". En Too Big to be Ignore Guidelines for Securing Sustainable Small-Scale Fisheries. MARES Series, Springer.

Food and Agriculture Organization. 2014. "The State of World Fisheries and Aquaculture Opportunities and Challenge". Roma: Food and Agriculture Organization of the United Nations.

Fujitani, Marie L, Eli P. Fenichel, Jorge Torre y Leah R. Gerber. 2012. "Implementation of A Marine Reserve Has A Rapid But Short-Lived Effect On Recreational Angler Use". Ecological Applications 22(2): 597-605.

Fulton, Stuart, Arturo Hernández-Velasco, Alvin Suarez-Castillo, Francisco Fernández-Rivera Melo, Mario Rojo, María Andrea SÁenz-Arroyo, Amy Hudson Weaver, Richard Cudney-Bueno, Fiorenza Micheli y Jorge Torre. En prensa 2017. "From Fishing Fish to Fishing Data: The Role of Artisanal Fishers in Conservation and Resource Management". En Navigating Small-Scale Fisheries Towards Viability and Sustainability: 
Experiences from Latin America and the Caribbean. MARES Series, Springer.

Gabriel-Morales, Josefina y José Luis Perez-Damian. 2006. "Crecimiento poblacional e instrumentos para la regulación ambiental de los asentamientos humanos en los municipios costeros de México". Gaceta Ecológica (79): 53-77.

Germain, Nathalie, Hans.J. Hartmann, Francisco Javier Fernández-Rivera Melo y Hector Reyes-Bonilla. 2015. "Ornamental Reef Fish Fisheries: New Indicators of Sustainability and Human Development at a Coastal Community Level". Ocean \& Coastal Management, vol. 104: 136-149.

Girardo, Cristina y Prudencio MochI. 2012. "Las organizaciones de la sociedad civil en México: modalidades del trabajo y el empleo en la prestación de servicios de proximidad y/o relacionales". Economía, Sociedad y Territorio XII(39): 333-357.

Harper, Sarah, Dirk Zeller, Melissa Hauzer, Daniel Pauly y Ussif Rashid Sumaila. 2013. "Women and Fisheries: Contribution to Food Security and Local Economies". Marine Policy 39(1): 56-63.

Herrmann Hans. 2004. "El papel de las organizaciones de la sociedad civil en el manejo costero en México". En El Manejo Costero en México, ed. Evelia Rivera-Arriga, Guillermo Villalobos-Zapata, Issac Azus-Adeathand, y Francisco Rosado May, 115-132. Campeche: Universidad Autónoma de Campeche, SEMARNaT, CETYS-Universidad, Universidad de Quintana Roo.

Instituto Mexicano para la Competitividad (imco). 2013. "Pesca ilegal: Una barrera a la competitividad, 2013". http:// cobi.org.mx/wp-content/uploads/2013/05/Pesca_Ilegal-web. pdf (Fecha de consulta: 10 de noviembre de 2016).

LaH, Kyung y Alberto Moya. "Aquatic Cocaine': Fish Bladders are Latest Mexican Smuggling Commodity". CNN. http://edition. cnn.com/2016/05/23/health/aquatic-cocaine-totoaba-basssmuggling/ (Fecha de consulta: 10 de noviembre de 2016).

Leslie, Heather M., Xavier Basurto, Mateja Nenadovicc, Leila Sievanena, Kyle C. Cavanaugh, Juan José Cota-Nieto, Brad E. Erisman, Elena Finkbeiner, Gustavo Hinojosa-Arango, 
Marcia Moreno-Báez, Sriniketh Nagavarapub, Sheila M.W. Reddy, Alexandra Sánchez-Rodríguez, Katherine Siegela, José Juan Ulibarria-Valenzuela, Amy Hudson Weaver y Octavio Aburto-Oropeza. 2015. "Operationalizing the SocialEcological Systems Framework to Assess Sustainability". Proceedings of the Natural History Academy of Sciences 112(19): 5979-5984.

López Sagástegui, Catalina, Octavio Aburto Oropeza, Marcia Moreno Báez, Ismael Mascareñas Osorio y Gustavo HinoJOSA Arango. 2014. "Ciencia ciudadana en el alto Golfo de California: abriendo camino en el manejo pesquero y la conservación". CONABIO. Biodiversitas (116): 1-6.

LuQue, Diana y Eduwiges Gómez. 2007. "La construcción de la Región del Golfo de California desde lo ambiental y lo indígena”. Rambihai 3(1): 83-116.

Mancha-Cisneros, Maria del Mar, Alvin N. Suárez Castillo, Jorge Torre, John M. Anderies y Leah R. Gerber. 2018. “The Role of Stakeholder Perceptions and Institutions for Marine Reserve Efficacy in the Midriff Islands Region, Gulf of California, Mexico". Ocean and Coastal Management. DOI.org/10.1016/j. ocecoaman.2018.01.024.

McCay, Bonnie J., Fiorenza Micheli, Germán Ponce-Díaz, Grant Murray, Geoff Shester, Saudiel Ramirez-Sanchez y Wendy Weisman. 2014. "Cooperatives, Concessions, and CoManagement on the Pacific Coast of Mexico". Marine Policy, vol. 44: 49-59.

McGuire, Tomas y Gloria Ciria Valdez-Gardea. 1997. "Endangered species and precarious lives in the Upper Gulf of California". Culture and Agriculture 19(3): 101-107.

MezA, Alejandra, Jorge Torre, Cesar Moreno, Mario Rojo, y Ana Luisa Figueroa. "Monitoreo de usos humanos de la Reserva de la Biosfera Isla San Pedro Mártir." IV Congreso Nacional de Áreas Naturales Protegidas en México, San Luis Potosí, 2009.

Micheli, Fiorenza, María Andrea Sáenz-Arroyo, Ashley GreenLEY, Leonardo Vázquez, Antonio Espinoza-Montes, Marisa Rossetto y Guilio De Leo. 2012. "Evidence that marine reser- 
ves enhance resilience to climatic impacts". PloS ONE 7(7): e40832. doi:10.1371/journal.pone.0040832.

Moreno-Báez, Marcia, Barron J. Orr, Richard Cudney-Bueno, y William W. Shaw. 2010. "Using Fishers' Local Knowledge to Aid Management at Regional Scales: Spatial Distribution of Small-Scale Fisheries in the Northern Gulf of California, Mexico". Bulletin of Marine Science 86(2): 339-353.

Moreno-BÁez, Marcia, Richard Cudney-Bueno, Barron J. Orr, William Shaw, Tad Pfister, Jorge Torre, Rene Loaiza y Mario Rojo. 2102. "Integrating the Spatial and Temporal Dimension of Fishing Activities for Management in the Northern Gulf of California, Mexico". Ocean \& Coastal Management, vol. 55: 111-127. Munguia-Vega, Adrian, Jorge Torre, Peggy Turk-Boyer, Silvio Guido Marinone, Miguel Fernando Lavín, Tad Pfister, William Shaw, Gustavo Danemann, Peter Raimondi, Alejandro Castillo-López, Ana Cinti, Jennifer N. Duberstein, Marcia Moreno-Báez, Mario Rojo, Gaspa r Soria, Laura SánchezVelasco, Hem Nalini Morzaria-Luna, Luis Bourillón, Kirsten Rowell y Richard Cudney-Bueno. 2015. "PANGAS: An Interdisciplinary Ecosystem-Based Research Framework for Small-Scale Fisheries in the Northern Gulf of California”. Journal of the Southwest 57(2-3): 337-390.

Murillo, Rosario y Amanda LejBowicz. 2014. Guia para la concepción y edificación del pueblo costero sustentable en Isla Magdalena, Baja California Sur, México. Comunidad y Biodiversidad, A.C. http://cobi.org.mx/wp-content/uploads/2014/12/2014guia-pueblo-costero-sustentable-coBı.pdf

Ostrom, Elinor. El gobierno de los bienes comunes. La evolución de las instituciones de acción colectiva. 2000. México: Universidad Nacional Autónoma de México, Centro Regional de Investigaciones Multidisciplinarias, Fondo de Cultura Económica.

Sánchez-Bajo, Claudia y Bruno Roelants. 2013. "Natividad Island Divers' and Fishermen's Cooperative, Mexico: Managing Natural Resources to Generate Wealth". En Capital and the debt trap: Learning from cooperatives in the global crisis. Londres: Palgrave Macmillan. 
Sánchez-Ibarra, Cesar, Diana. M. Bermúdez-García, Juan E. Bezaury-Creel, Cristina Lasch-Thaler, Natalie RodríguezDowdell, Nirari Cárdenas-Torres, Susana Rojas-González de Castilla, Anne Gondor, eds. 2013. Plan de acción para la conservación y aprovechamiento sustentable de la biodiversidad terrestre y marina de la región Golfo de California y Pacifico SudCaliforniano. México: Comisión Nacional de Áreas Naturales Protegidas, The Nature Conservancy, Fondo Mexicano para la Conservación de la Naturaleza, A.C.

Secretaría de Medio Ambiente y Recursos Naturales (semarNAT). 2012. Plan de manejo tipo para peces marinos de ornato. México: SEMARNAT.

Smith, Martin, Cathy A. Roheim, Larry B. Crowder, Benjamin S. Halpern, Mary Turnipseed, James L. Anderson, Frank Asche, Luis Bourillón, Atle G. Guttormsen, Ahmed Khan, Lisa A. Liguori, Aaron McNevin, Mary I. O’Connor, Dale Squires, Peter Tyedmers, Carrie Brownstein, Kristin Carden, Dane H. Klinger, Raphael Sagarin y Kimberly A. Selkoe. 2010. "Sustainability and Global Seafood". Science 327(5967): 784-786.

Torre, Jorge y Mario Rojo. 2011. Reserva de la Biosfera Isla San Pedro Mártir: comunidad, monitoreo y oportunidades. México: Reporte Anual Fondo Mexicano para la Conservación de la Naturaleza.

Villaseñor-Derbez, Juan Carlos, Arturo Hernández-Velasco, Maite Erauskin, Stuart Fulton, Juan José Cota-Nieto, Jorge Torre, Sharon Herzka-Llona, Octavio Aburto-Oropeza. 2015. "Effects of Short-Term Marine Reserves on Grouper and Bass Populations". DataMares. InteractiveResource. http:// dx.doi.org/10.13022/M33K52

Torre, Jorge, Eduardo Rolón, María José Espinosa y Stuart FuLTON. 2016. "Zonas de recuperación marina". En Análisis y propuestas para la conservación de la Biodiversidad en México 1995-2017, comp. Manuel Llano y Humberto Fuentes, 61-68. México: Fondo Mexicano para la Conservación de la Naturaleza, http://www.biodiversidad2016.org 
VINCE, Erik. 2017. "Requiem for the Vaquita. What the Demise of a Small Mexican Porpoise Tell us about Extinction in the 21 Century". Scientific American 317(2) (agosto): 36-45.

Wallace, Marshall. 2015. From Principle to Practice. A User's Guide to Do No Harm. CDA Collaborative Learning Projects. 